\title{
Non-invasive Diagnosis of Fibrosis in Non-alcoholic Fatty Liver Disease
}

\author{
Anil Arora, Praveen Sharma \\ Departments of Gastroenterology and Hepatology, Sir Ganga Ram Hospital, New Delhi, India
}

\begin{abstract}
Non-alcoholic fatty liver disease (NAFLD) is the most common chronic liver disease in developed as well as in developing countries. Its prevalence continues to rise currently affecting approximately $20-30 \%$ of adults and $10 \%$ of children in the United States. Non-alcoholic fatty liver disease represents a wide spectrum of conditions ranging from fatty liver, which in general follows a benign non-progressive clinical course, to non-alcoholic steatohepatitis (NASH), a more serious form of NAFLD that may progress to cirrhosis and end-stage liver disease. Liver biopsy remains the gold standard for evaluating the degree of hepatic necroinflammation and fibrosis; however, several non-invasive investigations, such as serum biomarkers, have been developed to establish the diagnosis and also to evaluate treatment response. There has been a substantial development of non-invasive risk scores, biomarker panels, and radiological modalities to identify at risk patients with NAFLD without recourse to liver biopsy on a routine basis. Examples include combination of serum markers like NAFLD fibrosis score (NFS), BARD score, fibrometer, FIB4, and non-invasive tools like fibroscan which assess fibrosis in patients with NAFLD. Other markers of fibrosis that have been evaluated include high-sensitivity C-reactive protein, plasma pentraxin 3, interleukin-6, and cytokeratin-18. This review focuses on the methods currently available in daily clinical practice in hepatology and touches briefly on the potential future markers under investigation. (J Clin EXP HePATOL 2012;2:145-155)
\end{abstract}

$\mathrm{N}$ on-alcoholic fatty liver disease (NAFLD) encompasses a wide spectrum of conditions associated with over accumulation of fat in the liver which range from benign fatty liver to an intermediate stage of non-alcoholic steatohepatitis (NASH) leading to advanced fibrosis and cirrhosis. Together, NASH and hepatic steatosis collectively affect between $20 \%$ and $30 \%$ of people in developing and developed countries, respectively, making NAFLD the commonest liver ailment in the world. ${ }^{1}$ Nonalcoholic steatohepatitis, the more advanced and clinically important form of NAFLD, is less common, with an

Keywords: Fibrosis, NAFLD, NASH

Received: 24.03.2012; Accepted: 24.05.2012

Address for correspondence: Dr. Anil Arora, Department of Gastroenterology and Hepatology, Sir Ganga Ram Hospital, New Delhi, India

E-mail: dranilarora50@gmail.com

Abbreviations: ALP: alkaline phosphatise; ALT: alanine aminotransferase; APRI: AST to platelet ratio index; AST: aspartate aminotransferase; AUCs: area under the curves; AUROC: area under the receiver-operating characteristics curve; BMI: body mass index; CCL2: CC-chemokine ligand-2; CI: confidence interval; CT: computed tomography; ECM: extracellular matrix; ELF: European liver fibrosis; FT: FibroTest; GGT: gammaglutamyl-transpeptidase; HA: hyaluronic acid; HTGC: hepatic triglyceride levels; LSM: liver stiffness; MRE: magnetic resonance elastography; MRI: magnetic resonance imaging; MRS: magnetic resonance spectroscopy; NAFL: non-alcoholic fatty liver; NAFLD: non-alcoholic fatty liver disease; NASH: non-alcoholic steatohepatitis; NFS: NAFLD fibrosis score; NPV: negative-predictive value; OR: odds ratio; PPV: positive-predictive value; SF: serum ferritin; SPEA: Serum prolidase enzyme activity; TE: transient elastography; TIMP1: tissue inhibitor of metalloproteinase 1; TNF: tumor necrosis factor; TRX: thioredoxin; US: ultrasonography doi: 10.1016/S0973-6883(12)60103-0 estimated prevalence of $2-3 \%$ in the general population and $16 \%$ and $37 \%$ in the morbidly obese individuals., Another issue of concern is that NAFLD now affects $3 \%$ of the general pediatric population, rising to $53 \%$ in obese children $^{4,5}$ with considerable implications for future liver disease burden. The most common benign form of NAFLD is fatty liver and represents about $80-90 \%$ of cases, whereas $\mathrm{NASH}$ is a potentially serious condition associated with a significant increase in the overall and liver-related morbidity and mortality. ${ }^{6,7}$

\section{NATURAL HISTORY OF NON-ALCOHOLIC FATTY LIVER DISEASE}

The short-term prognosis of NAFLD is largely excellent, but long-term prognosis depends crucially on histological stage at presentation. ${ }^{8,9}$ In patients with bland steatosis, two studies have reported either nil ${ }^{10}$ or minimal progression $^{11}$ to advanced disease over a median of 11.5 and 16.7 years, respectively. For those with NASH on baseline liver biopsy, $11 \%$ went on to develop cirrhosis and $40 \%$ of patients die from any cause within 15 years of which $7 \%$ are from liver-related complications, especially in those with advanced fibrosis or cirrhosis. Studies with liver biopsy on follow-up have also prospectively evaluated the risk of fibrosis progression over time. ${ }^{12,13}$ One hundred and three patients underwent two liver biopsies 1-21 years apart: Baseline low fibrosis stage, diabetes, and greater body mass index (BMI) were independently associated with fibrosis progression. ${ }^{14}$ In another study, 70 patients underwent two liver biopsies 10-16 years apart; progression of 
fibrosis stage occurred in $41 \%$ and was associated with diabetes, weight gain, and increased insulin resistance. ${ }^{15} \mathrm{An}$ important issue in patients with NAFLD is to differentiate NASH from simple steatosis as NASH patients are at sustained risk of developing complications of chronic liver disease (CLD), such as cirrhosis, liver failure, and hepatocellular carcinoma.

The diagnosis of NASH, which is characterized by necroinflammation, ballooning degeneration, and fibrosis, is essentially based on histological examination by liver biopsy. The liver biopsy removes only about $1 / 50,000$ th of the liver which is a painful and invasive procedure with potentially life-threatening complications. ${ }^{16,17}$ Complicating the ability of a liver biopsy to diagnose NASH is interobserver and intra-observer variabilities between histopathologists. Grading of steatosis is reliable with Kappa statistics for intra-observer and inter-observer variabilities between $0.74-0.98$ and 0.79 , respectively. However, fibrosis staging, lobular inflammation, and ballooning interpretation have relatively high intra-observer and inter-observer variabilities. ${ }^{18-21}$ In addition, given the numbers of patients with NAFLD, the use of liver biopsy is clinically and financially impractical. These limitations have stimulated the search for new non-invasive approaches for diagnosis of fibrosis in liver. Ideally, a non-invasive marker of liver fibrosis should be liver-specific, easy to perform, reliable, reproducible, and inexpensive. It should, in addition, be accurate not only for the staging of fibrosis, but also for monitoring disease progression. For all of these very reasons, there is an urgent need to develop and validate simple, reproducible, non-invasive tests that accurately distinguish NASH from non-alcoholic fatty liver (NAFL) or simple steatosis and determine if possible both the stage and grade of the disease.

\section{LABORATORY TESTS}

Most patients with NAFLD, including adults and children with either NAFL or NASH, are asymptomatic at presentation. The features more consistently found to be associated with disease severity include obesity, older age, diabetes, and hypertension. Laboratory tests that are routinely included in the evaluation of patients with suspected NAFLD include a serum panel of liver tests (alanine aminotransferase [ALT], aspartate aminotransferase [AST], alkaline phosphatase [ALP], gamma-glutamyl-transpeptidase [GGT], and albumin), prothrombin time, and complete blood count.

Elevated serum ALT and AST levels are the primary abnormality seen in patients with NAFLD and tend to be higher in patients with NASH as compared with NAFL. However, ALT levels are seldom higher than five times the upper limit of normal, and typically fluctuate with normal levels seen in more than two-thirds of patients with NASH over a period of time. Mofrad et $\mathrm{al}^{22}$ demonstrated that the one can find the entire histological spectrum of NAFLD with normal ALT values. In a similar study by Fracanzani et $\mathrm{al}^{23} 455$ patients with NAFLD were divided in two groups according to their serum ALT levels. They compared clinical and histological features of patients with and without increased serum ALT. Non-alcoholic steatohepatitis was diagnosed in $62 \%$ and $74 \%$ of patients with normal or increased ALT levels, respectively. There were no significant differences in advanced fibrosis between the two groups with or without raised serum ALT, underlying the need for liver biopsy for a definitive diagnosis and staging of fibrosis in NASH.

Among other routine laboratory tests, a reversal of the AST/ALT ratio (AAR) to $>1$ had been consistently reported to predict the presence of more advanced fibrosis. This observation is also true in a variety of other CLDs. ${ }^{24}$ In addition to its utility as an individual marker, the AAR is also a component of several other fibrosis scoring systems including the NFS and BARD score (BMI, AAR, and the presence of type 2 diabetes mellitus). ${ }^{25}$ Despite its simplicity, using a cut-off of $0.8 \mathrm{McPherson}$ et $\mathrm{al}^{26}$ demonstrated a sensitivity (74\%), specificity (78\%), and negative-predictive value (NPV) of $93 \%$ for the diagnosis of advanced fibrosis in NAFLD using the AAR.

Increased serum GGT level has also been shown to be associated with advanced fibrosis in NAFLD, with a study of 50 patients with NAFLD demonstrating an area under the receiver-operating characteristics curve (AUROC) of 0.74 for the prediction of advanced fibrosis. Using a cut-off serum GGT value of $96.5 \mathrm{U} / \mathrm{L}$, GGT predicted advanced fibrosis with $83 \%$ sensitivity and $69 \%$ specificity. ${ }^{27}$

The AST to platelet ratio index (APRI) was originally developed for use in chronic hepatitis $\mathrm{C}$. In a recent metaanalysis, the summary area under the curves (AUCs) of the APRI for significant fibrosis and cirrhosis were 0.76 (95\% confidence interval [CI], 0.74-0.79) and 0.82 (95\% CI, 0.79-0.86), respectively, for hepatitis $\mathrm{C}$ related fibrosis. For significant fibrosis, an APRI threshold of 0.5 was $81 \%$ sensitive and $50 \%$ specific. ${ }^{28}$ Its utility in NAFLD has since been studied by a number of researchers. Using this score, Cales et $\mathrm{al}^{29}$ demonstrated an AUROC of 0.866 for significant fibrosis, 0.861 for severe fibrosis, and 0.842 for cirrhosis in a study of 235 NAFLD subjects. However, significantly lower values were obtained in other studies, where AUROCs of 0.564 for significant fibrosis, 0.568 for advanced fibrosis, and 0.786 for predicting cirrhosis were demonstrated. ${ }^{30-32}$

Serum ferritin (SF) levels are commonly elevated in patients with NAFLD because of systemic inflammation, increased iron stores, or both. Manousou et al ${ }^{33}$ studies 111 patients and found on multivariate regression that diabetes, SF concentrations, BMI, and AST were independently associated with NASH. In a recent study by Kowdley et $\mathrm{al}^{34}$ who studied SF in 628 adult patients with NAFLD 
(age, $\geq 18$ years) with biopsy-proven NAFLD and an SF measurement within 6 months of their liver biopsy, it was concluded that SF $>1.5 \times$ upper limit of normal (ULN) is associated with hepatic iron deposition, a diagnosis of $\mathrm{NASH}$, and worsened histologic activity and is an independent predictor of advanced hepatic fibrosis among patients with NAFLD. Furthermore, elevated SF is independently associated with higher NAS, even among patients without hepatic iron deposition.

Laboratory signs of advanced liver disease, such as hyperbilirubinemia, hypoalbuminemia, and abnormal prothrombin time, are seen only in cases associated with cirrhosis.

\section{IMAGING TECHNIQUES}

Non-invasive techniques such as ultrasound, computed tomography (CT), magnetic resonance imaging (MRI), and proton magnetic resonance spectroscopy (1H-MRS) can detect hepatic steatosis, but cannot reliably distinguish simple steatosis from NASH.

\section{ULTRASOUND}

Ultrasonography (US) is currently the preferred method for screening asymptomatic patients with elevated liver enzymes and suspected NAFLD. Ultrasonography findings of fatty liver include hepatomegaly, diffuse increase in echogenicity of the liver parenchyma, and vascular blunting. The sensitivity of US in detecting steatosis varies between $60 \%$ and $94 \%,{ }^{35}$ and also varies depending on steatosis degree. Sensitivity is very low when the degree of steatosis is $<30 \%$; this technique has important limitations. First, it is operator-dependent and subject to significant intra- and inter-observer variability, ${ }^{36-38}$ second, US does not provide quantitative information of the degree of lipid accumulation. Another limitation is its inability to differentiate simple steatosis from steatohepatitis as both of them have similar appearance on ultrasound.

\section{DIFFERENTIATION OF NON-ALCOHOLIC FATTY LIVER DISEASE FROM NON- ALCOHOLIC STEATOHEPATITIS}

A simple non-invasive and easy to perform parameter is longitudinal diameter of the spleen. As the study of Tarantino et $\mathrm{al}^{39}$ recently showed, spleen diameter could differentiate between NAFLD and NASH better than both IL-6 and vascular endothelial growth factor, with values $>116 \mathrm{~mm}$ predicting NASH. Iijima et $\mathrm{al}^{40}$ evaluated the utility of contrast ultrasound with levovist for the diagnosis of NASH. Levovist, an ultrasound contrast agent, was injected intravenously and the liver was scanned at different time intervals. They found a statistically significant decrease of signal intensity in NASH, when compared with NAFLD, due to reduced uptake of levovist mediated by cell injury. However, larger studies are needed to establish the role of contrast US in the diagnosis of NASH.

\section{COMPUTED TOMOGRAPHY}

Non-contrast-enhanced CT is the most accurate CT technique to detect and characterize hepatic steatosis. ${ }^{41}$ The CT diagnosis of hepatic steatosis is made by measuring the difference in liver and spleen attenuation values in Hounsfield units. The density of the liver, as visualized by CT, decreases as the severity of steatosis increases. Computed tomography can also visualize splenomegaly as a sign of portal hypertension, which is suggestive for advanced fibrosis in patients with NAFLD. In a study by Park et $\mathrm{al}^{42} 154$ candidates, including 104 men and 50 women, underwent unenhanced CT and US-guided liver biopsy on the same day. They concluded that diagnostic performance of unenhanced CT for quantitative assessment of macrovesicular steatosis is not clinically acceptable. Unenhanced CT, however, provides high performance in qualitative diagnosis of macrovesicular steatosis of $30 \%$ or greater. However, CT exposes subjects to ionizing radiation and cannot assess concurrent inflammation and fibrosis.

\section{MAGNETIC RESONANCE IMAGING AND PROTON MAGNETIC RESONANCE SPECTROSCOPY}

Magnetic resonance imaging provides an accurate and rapid assessment of hepatic steatosis to a lower limit of $3 \%$. Phase-contrast imaging correlates with the quantitative assessment of fatty infiltration across the entire range of liver diseases. A new MRI technique, proton MRS, measures the fat proton fraction and hepatic triglyceride levels (HTGC). The 95th percentile of HTGC in subjects (Dallas Heart Study) was $5.56 \%$, which corresponds to a HTGC of $55.6 \mathrm{mg} / \mathrm{g}$. Thus, MRS provides a sensitive, quantitative, non-invasive method to measure HTGC and, when applied to a large urban US population, revealed a strikingly high prevalence of hepatic steatosis. ${ }^{43}$ Reproducibility of (1) H-MRS to measure HTGC for 'between-weeks' measurements and in fatty livers is high, which is important for follow-up studies. ${ }^{44}$ Magnetic resonance spectroscopy characterizes metabolic processes involved in cellular regeneration, and thus it can evaluate the disease severity in NASH. An increased ATP/phosphate ratio might be a signal for progression to an advanced stage of fibrosis in NASH. Magnetic resonance spectroscopy is probably more accurate than previous imaging procedures for the diagnosis of NAFLD but it needs validation in large human studies. In a study by Guiu B et al, ${ }^{45}$ patients underwent 3-T single-voxel point-resolved (1) H MR spectroscopy of the liver (Couinaud segment VII) which was performed to calculate the liver fat fraction from the water $(4.7 \mathrm{ppm})$ and methylene (1.3 ppm) peaks, corrected for T1 and T2 decays. Liver fat fraction was also computed from tripleecho (consecutive in-phase, opposed-phase, and in-phase echo times) breath-hold spoiled gradient-echo sequence (flip angle, $20^{\circ}$ ), by estimating $\mathrm{T}^{*}$ and relative signal 
intensity loss between in- and opposed-phase values, corrected for $\mathrm{T} 2 *$ decay. They concluded that a breath-hold triple-echo gradient-echo sequence with a low flip angle and correction for $\mathrm{T} 2 *$ decay is accurate for quantifying fat in segment VII of the liver. Given its excellent correlation and concordance with (1)H MR spectroscopy, this triple-echo sequence could replace (1)H MR spectroscopy in longitudinal studies if and when validated in large number of patients with NAFLD. ${ }^{45,46}$

Both computerized tomographic scanning and, in particular, MRI seem to be more sensitive techniques for quantification of steatosis. However, none of these imaging techniques have sufficient sensitivity and specificity for staging the disease and cannot distinguish between simple steatosis and NASH with or without fibrosis.

\section{MAGNETIC RESONANCE ELASTOGRAPHY}

An MR equivalent of transient elastography (TE) has recently demonstrated excellent diagnostic accuracy with sensitivity and specificity of $98 \%$ and $99 \%$, respectively, for detecting all grades of fibrosis. ${ }^{40}$ In a prospective study by Huwart et al, ${ }^{47}$ prospective blind comparison of magnetic resonance elastography (MRE), ultrasound elastography, and APRI in a 141 consecutive series of patients who underwent liver biopsy for CLD was studied. The areas under the receiver-operating characteristic curves of MRE ( 0.994 for $\mathrm{F} \geq 2 ; 0.985$ for $\mathrm{F} \geq 3 ; 0.998$ for $\mathrm{F}=4$ ) were larger $(P<0.05)$ than those of ultrasound elasticity, APRI, and the combination of ultrasound elasticity and APRI. Magnetic resonance elastography was also associated with a higher technical success rate than US elastography and hepatic stiffness did not appear to be affected by steatosis using this technique which had been a previous concern. However, this technique remains experimental at this time.

\section{FIBROSCAN}

Transient elastography (Fibroscan, Echosens, Paris, France) is a non-invasive method of assessing liver fibrosis which can be performed at the bedside or in the outpatient clinic. It employs ultrasound-based technology to measure liver stiffness (LSM), and has been validated for use mainly in patients with chronic hepatitis $\mathrm{C}$ and chronic hepatitis B. ${ }^{48,49}$ However, recent studies had shown good results in patients with NAFLD. ${ }^{50,51}$ Failure to obtain a reading occurs in only $5 \%$ of cases, but is more common in obese patients which has so far limited its use in the NAFLD cohort, although a recently introduced XL probe may reduce this problem. ${ }^{52}$ Although fibroscan is less well validated in NAFLD, liver stiffness measurements in 67 NAFLD patients (mean [SD] age, 50.4 [3.3] years) in whom the diagnosis had been confirmed by liver biopsy and the severity of fibrosis had been scored according to Brunt. The median liver stiffness values (with 95\% CIs) were F0, 4.907 (4.417-5.396) kPa; F1, 6.142 (5.582-6.702) kPa; F2,
7.894 (6.384-9.404) kPa; F3, 11.027 (8.555-13.500) kPa; $\mathrm{F} 4,26.960(17.705-36.215) \mathrm{kPa}$. Stepwise increase in liver stiffness with increasing histological fibrosis was demonstrated in this study of NAFLD patients. ${ }^{53}$ In a recent study, 246 consecutive patients from two ethnic groups had successful liver stiffness measurement and satisfactory liver biopsy specimens. The AUROC of TE for F3 or higher and F4 disease was 0.93 and 0.95 , respectively, and was significantly higher than that of the AAR, APRI, FIB-4, BARD, and NFSs (AUROC ranged from 0.62 to $0.81, P<0.05$ for all comparisons). At a cut-off value of $7.9 \mathrm{kPa}$, the sensitivity, specificity, and positive- and NPVs for F3 or greater disease were $91 \%, 75 \%, 52 \%$, and $97 \%$, respectively. With high NPV and modest positive-predictive value (PPV), TE is useful as a screening test to exclude advanced fibrosis. Liver biopsy may be considered in NAFLD patients with liver stiffness of at least $7.9 \mathrm{kPa}^{51}$ In a recent meta-analyses for NASH with advanced fibrosis, pooled AUROC, sensitivity and specificity of NFS, and fibroscan are 0.85 (0.80-0.93), 0.90 (0.82-0.99), 0.97 (0.94-0.99) and 0.94 (0.90-0.99), 0.94 (0.88-0.99) and 0.95 (0.89-0.99), respectively. ${ }^{54}$ These values are different from patients with HCV-related fibrosis as in a study by Castéra et $\mathrm{al}^{55}$ where fibroscan values ranged from $2.4 \mathrm{kPa}$ to $75.4 \mathrm{kPa}$ (median, $7.4 \mathrm{kPa}$ ). Cut-off values were $7.1 \mathrm{kPa}$ for $\mathrm{F} \geq 2,9.5 \mathrm{kPa}$ for $\mathrm{F} \geq 3$, and $12.5 \mathrm{kPa}$ for $\mathrm{F}=4$. Although further studies will undoubtedly add to the current evidence base, fibroscan has now been validated in NAFLD, and represents a useful tool for rapid, non-invasive assessment of liver fibrosis and determining the need for biopsy. Fibroscan evaluates liver stiffness, which is related to fibrosis, inflammation, and portal hypertension. Therefore, fibroscan values have to be interpreted according to clinical, biological, and morphological data.

\section{Markers of Fibrosis}

As individual clinical or biochemical markers are not sufficiently accurate to predict the presence of NASH, multiple parameters have been combined into mathematical models to produce diagnostic algorithms to facilitate the non-invasive assessment of NAFLD and differentiation between different stages of NAFLD. Such scoring systems have generally demonstrated greater utility in the detection of advanced fibrosis than intermediate and early stages of fibrosis, a group which potentially is more likely to benefit from therapeutic interventions. ${ }^{56}$

The BARD score is a simple scoring system designed to identify NAFLD patients with a low-risk of advanced disease. It combines three variables of BMI, AAR, and the presence of diabetes into a weighted sum (BMI $>28=1$ point, $\mathrm{AAR}$ of $>0.8=2$ points, $\mathrm{DM}=1$ point), to generate a score from 0 to 4 . In the original study, a score of 2-4 was shown to be associated with an odds ratio (OR) for advanced fibrosis of 17 and a NPV of $96 \%{ }^{49}$ The BARD score was also validated in a Polish NAFLD cohort, where 
an NPV of $97 \%$ was demonstrated, but appeared less useful in a Japanese cohort, where the AUROC was 0.73 with NPV $77 \% .57,58$

The FIB-4 test combines age with three standard biochemical values (platelets, ALT, and AST) to assess fibrosis. In NAFLD, FIB-4 has demonstrated similar results to the AAR where, using a cut-off of 1.3 , its sensitivity $85 \%$, specificity $65 \%$, and NPV of $95 \%$ were demonstrated for the diagnosis of advanced fibrosis. In another study in 541 patients with NAFLD, FIB-4 had the highest PPV and NPV of $80 \%$ and $90 \%$, respectively, for the diagnosis of advanced fibrosis. ${ }^{58-60}$ In a study by Sumida et $\mathrm{al}^{61}$ in Japanese cohort of 576 patients with biopsy-proven NAFLD, FIB4 index was superior to those for the other scoring systems for differentiating between advanced and mild fibrosis.

FibroMeter is a panel of serum markers which was originally developed for staging fibrosis in chronic HCV. ${ }^{62,63}$ However, fibrometer NAFLD has since been developed which has shown good diagnostic accuracy in staging NASH-related fibrosis. This panel combines seven variables (age, weight, fasting glucose, AST, ALT, ferritin, and platelet count), and in a study of 235 NAFLD patients the highest accuracy $91 \%$ for significant fibrosis was obtained with the fibrometer whose (i) AUROC (0.943) was significantly higher than those of NAFLD fibrosis score (NFSA, $0.884, P=0.008)$ and APRI $(0.866, P<0.0001$ vs NFSA $)$ in the whole population. ${ }^{29}$

The NFS is a panel comprising six variables of age, hyperglycemia, BMI, platelet count, albumin, and AAR, which was constructed using a large panel of 733 biopsyproven NAFLD. In a recent meta-analyses, NFS achieved pooled sensitivity and specificity of, $0.90(0.82-0.99)$ and 0.97 (0.94-0.99) for the identification of NASH with advanced fibrosis. ${ }^{54}$ The NFS thus facilitates the identification of NAFLD patients with more advanced disease who require ongoing follow-up, and considerably reduces the requirement for liver biopsy in the minority of patients with an indeterminate score. In a recent study by Armstrong et $\mathrm{al}^{64}$ in a primary-care cohort data from 1118 adult patients were analyzed. The cause of abnormal liver functional tests was identified in 55\% (614/1118) of subjects, with NAFLD (26.4\%; 295/1118) and alcohol excess (25.3\%; 282/1118) accounting for the majority. A high NFS $(>0.676)$ suggesting the presence of advanced liver fibrosis was found in $7.6 \%$ of NAFLD subjects, whereas $57.2 \%$ of NAFLD patients had a low NFS $(<-1.455)$ allowing advanced fibrosis to be confidently excluded. Of these various algorithms, FIB-4 and the NFS have been validated most widely and have demonstrated superior test characteristics.

Liver fibrosis represents the most worrisome histopathological feature in patients with NAFLD, because it suggests a more severe and progressive liver damage. Thus, as in other liver diseases, accurate assessment of the extent of fibrosis is essential for patients with NAFLD. Several groups have developed various markers using a panel of markers of clinical and biochemical tests to generate clinical models of fibrosis, whereas others focused on specific markers of fibrosis either in isolation or in multicomponent tests.

Ratziu et al ${ }^{65}$ combined four clinical variables to generate the BAAT score: $\mathrm{BMI}\left(\geq 28 \mathrm{Kg} / \mathrm{m}^{2}\right)$, age ( $\geq 50$ years), ALT ( $\geq 2$ normal), and serum triglycerides $(\geq 1.7 \mathrm{mmol} / \mathrm{L})$. The presence of each variable gives 1 point in the combined score. Whereas a total score of 0 or 1 had a NPV of $100 \%$ for fibrosis, a high total score of 4 gave a sensitivity of $14 \%$ and a specificity of $100 \%$ for the detection of septal fibrosis.

FibroTest (FT) is another validated marker for the quantitative assessment of fibrosis in NAFLD, alcoholic liver disease (ALD), and chronic viral hepatitis. In a metaanalysis by Shaheen et al, ${ }^{66} 12$ studies were identified, 9 for FT $(n=1679)$, and 4 for fibroscan $(n=546)$. In heterogeneous analyses for significant fibrosis, the AUCs for FT and fibroscan were 0.81 (95\% CI 0.78-84) and 0.83 (0.03$1.00)$, respectively. At a threshold of approximately 0.60 , the sensitivity and specificity of the FT were $47 \%$ (35-59\%) and $90 \%$ (87-92\%), respectively. Combining five biochemical markers of haptoglobin, a2-macroglobulin, apolipoprotein A1, total bilirubin, and GGT, corrected for age and gender, a mean standardized AUROC of 0.84 for advanced fibrosis in patients with NAFLD was demonstrated using FT in one meta-analyses in which a total of 30 studies were included which pooled 6378 subjects with both FT and biopsy (3501 HCV, 1457 HBV, 267 NAFLD, 429 ALD, and 724 mixed). The AUROC for the diagnosis of the intermediate adjacent stages F2 vs F1 $(0.66 ; 0.63-0.68, n=2055)$ did not differ from that of the extreme stages F3 vs F4 (0.69; $0.65-0.72, n=817)$ or $\mathrm{F} 1$ vs F0 $(0.62 ; 0.59-0.65, n=1788) .{ }^{67}$ The most frequent causes of FT failure include Gilbert's syndrome, cholestasis, and acute inflammation, which result in increases in bilirubin and haptoglobulin, respectively, and an abnormal apolipoprotein A1 concentration.

\section{FibroMAX}

Among the non-invasive alternatives to liver biopsy, several studies have demonstrated the predictive value and a better benefit-to-risk ratio than liver biopsy of five combinations of simple serum biochemical markers (the super combination being FibroMAX (BioPredictive, Paris, France) in patients at risk of CLDs: FT (BioPredictive) for the quantitative assessment of fibrosis; SteatoTest (BioPredictive) for the quantitative assessment of steatosis; ActiTest (BioPredictive) for the quantitative assessment of necroinflammatory activity in chronic viral hepatitis $\mathrm{C}$ and $\mathrm{B}$; NashTest (BioPredictive) for the categorical diagnosis of $\mathrm{NASH}$; and AshTest for the quantitative assessment of alcoholic steatohepatitis (also known in the USA as HCV-FibroSURE, HBV-FibroSURE, ASH-FibroSURE, and NASH-FibroSURE; LabCorp, NC, USA). ${ }^{68,69}$ 


\section{Predictive Model for the Diagnosis of Non-alcoholic Steatohepatitis}

As individual clinical or biochemical markers are not sufficiently accurate to predict the presence of NASH, multiple parameters have been combined into mathematical models to produce various diagnostic algorithms (Table 1). Dixon et $\mathrm{al}^{70}$ first evaluated liver biopsies taken at laparoscopic obesity surgery in 105 consecutive patients. Twenty-six patients $(25 \%)$ were found to have NASH, and $11(42 \%)$ of these had advanced fibrosis. A raised index of insulin resistance (OR 9.3, 95\% CI 3.4-26), systemic hypertension (OR 5.2, 95\% CI 2-13.5), and raised ALA (OR 8.6, 95\% CI 3.1-23.5) was the independent predictor of NASH. A combination of two or three of these predictors allows a sensitivity of 0.8 and a specificity of 0.89 for NASH. Palekar et $\mathrm{al}^{71}$ generated a clinical model to distinguish $\mathrm{NASH}$ from simple steatosis by combining six different variables including age ( $>50$ years), female gender, AST $(>45 \mathrm{U} / \mathrm{L})$, BMI $\left(>30 \mathrm{Kg} / \mathrm{m}^{2}\right)$, and AAR $(>1)$, and serum hyaluronic acid (HA) $(>55 \mathrm{mg} / \mathrm{L})$. The AUC for this model was 0.76 . The presence of three or more of these factors had a sensitivity and specificity for NASH diagnosis of 74\% and $66 \%$, respectively. Gholam et $\mathrm{al}^{72}$ proposed a simplified model created using logistic regression analysis using only AST and diagnosis of diabetes, which was able to separate NASH from fatty liver with or without non-specific inflammation in bariatric surgery patients with similar accuracy as the panels described in previous studies.
Poynard et $\mathrm{al}^{73}$ developed a model (the NASHTest) using the combination of 13 variables including age, gender, height, weight, and serum levels of triglycerides, cholesterol, alfa2macroglobulin, apolipoprotein A1, haptoglobin, GGT, ALT, AST, and total bilirubin. One hundred and sixty patients with NAFLD were prospectively included in a training group, 97 were included in a multicenter validation group, and 383 controls. For the diagnosis of definite NASH defined by a NAFLD activity score of greater than 4, both sets had an AUC of 0.79. For the diagnosis of NASH as determined by the grading pathologist, the NASH test provided AUC in the training and validation sets of 0.78 and 0.69 , respectively.

Fibrosis is a dynamic process which may result in increased circulating levels of extracellular matrix (ECM) components. Several groups have used this reasoning to develop different blood tests using individual or a composite of ECM components. Suzuki et $\mathrm{al}^{74}$ determined the reliability of serum HA to predict the severity of hepatic fibrosis in 79 patients with histologically confirmed NAFLD. Serum HA was obtained at the time of liver biopsy. The logarithm of serum HA showed a significant positive correlation with the stage of fibrosis, and this association persisted after adjusting for age and serum albumin. The test was found to be useful for predicting severe fibrosis (stages 3-4) with AUC of 0.9 (95\% CI: 0.83, 0.97). The study could not evaluate the efficacy for moderate fibrosis (stage 2) due to the limited number of patients

Table 1 Serum markers of fibrosis in non-alcoholic fatty liver disease.

\begin{tabular}{|c|c|c|c|c|c|c|c|}
\hline Index & $\begin{array}{c}\text { No. of } \\
\text { patients }\end{array}$ & Factors & $\begin{array}{l}\text { Fibrosis } \\
\text { stage }\end{array}$ & Cut-off & PPV (\%) & NPV (\%) & AUC \\
\hline BAAT 65 & 93 & $\begin{array}{l}\text { Age, BMI, serum, ALT, } \\
\text { triglycerides }\end{array}$ & $\mathrm{F} \geq 2$ vs $\leq 1$ & 2 & 61 & 86 & 0.84 \\
\hline $\mathrm{OELF}^{76}$ & 61 & Age, HA, TIMP-1, PIIINP & $\mathrm{F} \geq 3 \mathrm{vs} \leq 2$ & $\begin{array}{l}0.375 \\
0.462\end{array}$ & $\begin{array}{l}80 \\
87\end{array}$ & $\begin{array}{l}98 \\
96\end{array}$ & 0.87 \\
\hline $\begin{array}{l}\text { ELF plus simple } \\
\text { Clinical marker }^{77}\end{array}$ & 192 & HA, TIMP-1, PIIINP plus NFS & $\begin{array}{l}F \geq 1 \\
F \geq 2 \\
F \geq 3\end{array}$ & $\begin{array}{l}-5.002 \\
-0.995 \\
-0.2826\end{array}$ & $\begin{array}{l}86 \\
94 \\
77\end{array}$ & $\begin{array}{l}66 \\
75 \\
99\end{array}$ & $\begin{array}{l}0.84 \\
0.93 \\
0.98\end{array}$ \\
\hline$H A^{82}$ & 79 & $\mathrm{HA}$ & $\mathrm{F} \geq 3$ vs $\leq 2$ & 46.1 & 51 & 96 & 0.92 \\
\hline $\mathrm{NFS}^{54}$ & $\begin{array}{l}480 \\
253\end{array}$ & $\begin{array}{l}\text { Hyperglycemia, BMI, platelet } \\
\text { count, albumin, AST:ALT, age }\end{array}$ & $\mathrm{F} \geq 3 \mathrm{vs} \leq 2$ & $\begin{array}{l}<-1.455 \\
>0.676\end{array}$ & $\begin{array}{l}56 \\
90\end{array}$ & $\begin{array}{l}93 \\
85\end{array}$ & $\begin{array}{l}0.88 \\
0.82\end{array}$ \\
\hline$N S^{86}$ & 112 & Type 4 collagen, HA & $\mathrm{F} \geq 3$ vs $\leq 2$ & $\begin{array}{l}\text { COLL } \geq 5 \text { or } \\
\mathrm{HA} \geq 50\end{array}$ & 66 & 95 & NS \\
\hline $\mathrm{FT}^{69}$ & $\begin{array}{l}170 \\
97\end{array}$ & $\begin{array}{l}\text { Total bilirubin, GGT, gender, } \\
\text { age, } \alpha 2 \text {-macroglobulin, } \\
\text { apolipoprotein, A1, haptoglobin }\end{array}$ & $\begin{array}{l}F \geq 2 \text { vs } \leq 1 \\
F \geq 3 \text { vs } \leq 2\end{array}$ & $\begin{array}{l}0.30 \\
0.70 \\
0.30 \\
0.70\end{array}$ & $\begin{array}{l}54 \\
98 \\
71 \\
97\end{array}$ & $\begin{array}{l}90 \\
76 \\
98 \\
89\end{array}$ & $\begin{array}{l}0.81 \\
0.88\end{array}$ \\
\hline BARD $^{56}$ & $\begin{array}{l}827 \\
160\end{array}$ & Diabetes mellitus, BMI, ALT:AST & $F \geq 3$ & $2-4$ & 43 & 96 & 0.81 \\
\hline
\end{tabular}

ALT: alanine aminotransferase; AST: aspartate aminotransferase; AUC: area under the curve; BMI: body mass index; ELF: enhanced liver fibrosis; FT: FibroTest; GGT: gamma-glutamyl transpeptidase; HA: hyaluronan; NFS: NAFLD fibrosis score; NPV: negative-predictive value; NS: not significant; OELF: original European liver fibrosis; PIIINP: N-terminal propeptide of type III pro-collagen; PPV: positive-predictive value; TIMP: tissue inhibitors of metalloproteinases. 
with this stage of fibrosis and showed low accuracy for mild fibrosis.

The original european liver fibrosis (ELF) test is a panel of automated immunoassays to detect three markers of matrix turnover in serum: HA, tissue inhibitor of metalloproteinase 1 (TIMP1), and aminoterminal peptide of procollagen III (P3NP), used in combination with age. ${ }^{75}$ The simplified ELF panel excludes age but has a similar diagnostic performance. The addition of five simple markersBMI, presence of diabetes/impaired fasting glucose, AAR, platelets, and albumin - to the ELF test improved diagnostic accuracy further, with AUROCs of $0.98,0.93$, and 0.84 for the diagnosis of severe, moderate, and no fibrosis, respectively. ${ }^{76}$ The ELF panel may also represent a useful prognostic tool, with a 1-unit change in ELF score shown to be associated with a doubling of the odds of significant liver-related mortality or morbidity at a 6-year follow-up. ${ }^{77}$

\section{Biomarkers of Hepatocyte Apoptosis, Inflammation, and Oxidative Stress}

Apoptosis plays an important role in liver injury and disease progression in NAFLD. An increase in hepatocyte cell death by apoptosis is typically present in humans with NASH as well as in animal models of NASH but absent in those with simple steatosis. Another interesting approach is represented by the evaluation of plasma caspase-3generated cytokeratin-18 (CK-18) fragments, a biomarker of hepatocytes apoptosis. Levels of CK-18 fragments were able to identify patients with NASH as compared with those with bland steatosis with remarkably high specificity and acceptable sensitivity. Using alone $e^{78-80}$ or combination ${ }^{81}$ of markers which included cleaved and intact CK-18 levels, adiponectin, and resistin, a model with AUC values between 0.73 and 0.91 , was developed in a cohort of 101 patients. Subsequently, the same panel was reevaluated in another sample of 79 patients and had a lower AUC of 0.70. This led to the development of another model (the NASH diagnostic panel) consisting of diabetes, gender, BMI, triglyceride, CK-18 fragments, and total CK-18 levels. ${ }^{82}$ This new model had higher AUC levels than NASH diagnostics or CK-18 fragment levels in isolation; however, this model has not been validated in an independent sample.

Other potential fibrosis biomarkers include type VI collagen 7S domain and HA, with the latter also representing a constituent of the ELF test. In a cohort of 112 NAFLD subjects, these two biomarkers were able to exclude advanced fibrosis with AUROCs of 0.82 and 0.80, and NPVs of $84 \%$ and $78 \%$, respectively. These biomarkers also demonstrated PPVs of $86 \%$ and $92 \%$ and AUROCs of 0.83 and 0.80 for discriminating NASH from simple fatty liver. ${ }^{83}$

Other studies of HA in NAFLD using varying cut-off levels have demonstrated AUROCs of between 0.89 and 0.97 for detecting advanced fibrosis. Kaneda et $\mathrm{al}^{84}$ demonstrated HA to have an AUROC, NPV, sensitivity, and specificity of $0.97 \%, 100 \%, 100 \%$, and $89 \%$, respectively, for detecting severe fibrosis, with a lower AUROC of 0.87 demonstrated for type IV collagen. Lesmana and Yoneda et $\mathrm{al}^{85,86}$ also demonstrated the utility of levels of HA and type IV collagen to differentiate between mild (F12) and advanced fibrosis (F3-4), and in a separate study of 80 patients with NAFLD, the combination of HA with AST, AAR, age, gender, and BMI demonstrated an AUROC of 0.763 for distinguishing simple steatosis and NASH. ${ }^{71}$ In a small study of serum ECM components in 30 patients with NAFLD, serum laminin $>282 \mathrm{ng} / \mathrm{mL}$ was shown to have an accuracy of $87 \%$, sensitivity $82 \%$, specificity $89 \%$, PPV $82 \%$, and NPV $89 \%$ for identifying the presence of NASH with fibrosis. ${ }^{87}$

A growing body of evidence supports a central role for inflammation and inflammatory cytokines in the development of the metabolic syndrome and NAFLD. A cytokine imbalance, and in particular an increase in the tumor necrosis factor- $\alpha$ (TNF- $\alpha$ )/adiponectin ratio, may play an important role in the development of NASH. ${ }^{78}$ Two other inflammatory markers, interleukin-6 (IL-6) and highsensitive C-reactive protein (hs-CRP), which have been extensively studied as markers for insulin resistance, type- 2 diabetes, and cardiovascular disease, have been tested in patients with NAFLD. Hui et $\mathrm{al}^{78}$ quantified serum levels of both TNF- $\alpha$ and adiponectin in patients with biopsyproven NAFLD. Adiponectin levels were significantly lower and TNF- $\alpha$ levels were significantly higher in patients with NASH as compared with controls. However, when compared with simple steatosis, only adiponectin levels remained significantly lower in NASH patients, whereas no difference was noted in TNF- $\alpha$ levels between the two groups of patients. In another study, hs-CRP, IL-6, and several chemokines, including., were measured in 47 patients with biopsy-proven NAFLD as well as 30 agematched, sex-matched, and ethnicity-matched controls. Both IL- 6 and CCL2 but not hs-CRP were increased in patients with NAFLD as compared with controls. Moreover, only CCL2 levels were different between patients with NASH and those with simple steatosis. ${ }^{88}$ In a large cohort of patients $(n=120)$ in a study by Hui et al, there were significant associations between the hs-CRP levels and age $(r=0.2, P=0.01)$, BMI $(r=0.5, P=0.01)$, waist-to-hip ratio $(r=0.4, P=0.001)$, and insulin resistance by homeostasis model $(r=0.2, P=0.04)$. However, no relationship was discerned between the levels of hs-CRP and the grades of hepatic steatosis $(r=0.2, P=0.1)$, necroinflammation $(r=0.1$, $P=0.4)$, and fibrosis $(r=0.1, P=0.5)$. The mean $( \pm \mathrm{SD})$ hsCRP for NASH was $2.68 \pm 2.10 \mathrm{mg} / \mathrm{L}$, and $2.23 \pm 1.68 \mathrm{mg} / \mathrm{L}$ for simple steatosis $(P=0.4){ }^{89}$

Markers of stress and inflammation like IL- 6 and thioredoxin (TRX) which is stress-inducible thiol-containing protein may represent a clinically useful indicator of oxidative stress and found to be increased in patients with 
Table 2 Plasma candidate biomarkers for distinguishing simple steatosis from non-alcoholic steatohepatitis.

\begin{tabular}{|c|c|c|c|c|}
\hline Author & Markers & Functions & Results & Area under curve \\
\hline Hui et $\mathrm{al}^{78}$ & Adiponectin & $\begin{array}{l}\text { Adipose tissue metabolism/ } \\
\text { adipokines }\end{array}$ & $\begin{array}{l}\text { Reduced in NAFLD compared with } \\
\text { controls, lowest levels in NASH }\end{array}$ & 0.79 \\
\hline Lemoine et $\mathrm{al}^{79}$ & Leptin & $\begin{array}{l}\text { Adipose tissue metabolism/ } \\
\text { adipokines }\end{array}$ & $\begin{array}{l}\text { HOMA were correlated positively with } \\
\text { leptin levels }\end{array}$ & 0.82 \\
\hline Tarantino et al ${ }^{90}$ & IL-6 & Inflammation & Increased in patients with $\mathrm{NASH}$ & 0.817 \\
\hline Sumida et $\mathrm{al}^{91}$ & Thioredoxin & Stress-inducible protein & Increased in NASH & 0.785 \\
\hline Charlton et al ${ }^{93}$ & Dehydroepiandrosterone & Oxidative stress & Low circulating DHEA-S & 0.83 \\
\hline Feldstein et al ${ }^{80}$ & Cytokeratin-18 (CK-18) & Hepatocyte apoptosis & Increased in patients with NASH & 0.83 \\
\hline Yilmaz et al ${ }^{99}$ & sRage & $\begin{array}{l}\text { Glycation end products adipose } \\
\text { tissue metabolism }\end{array}$ & Reduced in NASH & 0.77 \\
\hline Kayadibi et al ${ }^{92}$ & $\begin{array}{l}\text { Serum prolidase enzyme } \\
\text { activity }\end{array}$ & Oxidative stress & Increased in NASH & 0.85 \\
\hline Kukla et al ${ }^{99}$ & Chemerin & $\begin{array}{l}\text { Adipose tissue metabolism/ } \\
\text { adipokines }\end{array}$ & Increased in NASH & - \\
\hline Alkhouri et al ${ }^{96}$ & Retiniol-binding protein 4 & $\begin{array}{l}\text { Adipose tissue metabolism/ } \\
\text { adipokines }\end{array}$ & Lower level in NASH & - \\
\hline Degertekin et $\mathrm{al}^{97}$ & Endothelin 1 & Hepatic fibrogenesis & Higher level in NASH & - \\
\hline Yilmaz et al ${ }^{98}$ & Angiopoietin-like protein 3 & Plasma triglyceride clearance & Higher level in NASH & - \\
\hline Tamimi et $\mathrm{al}^{94}$ & Soluble Fas & Hepatocyte apoptosis & Higher level in NASH & 0.79 \\
\hline
\end{tabular}

DHEA-S: dehydroepiandrosterone; NAFLD: non-alcoholic fatty liver disease; NASH: non-alcoholic steatohepatitis.

NASH. ${ }^{90,91}$ Serum prolidase enzyme activity (SPEA) catalyzes the final step of collagen breakdown by liberating free praline for collagen recycling, and is reported to be of hepatic origin which has also been shown to increase in patients with NASH..$^{92}$ Indicators of oxidative stress, ${ }^{90,93}$ apoptosis, ${ }^{94,95}$ and various adipokines ${ }^{96-98}$ have been investigated as surrogate markers of NASH in smaller studies which need more validation. However, most studies to date have been small with mixed results and it is not yet clear whether or not oxidative stress in the liver is accurately reflected in the serum (Table 2 ). ${ }^{99,100}$

\section{FUTURE AREAS FOR NON-INVASIVE TESTS}

As important progress is made in the elucidation of the pathogenesis of NAFLD, new rational non-invasive serum biomarkers that reflect the pathobiology of the disease, including markers of oxidative stress (OS), inflammation, apoptosis, and fibrosis, are being tested. All these markers are in the initial phases of investigation, and the clinical utility of these tests remains to be determined. ${ }^{101}$ Recent advances in the field of proteomics have provided us with powerful tools for discovering biomarkers in the field of NAFLD. Among the different technologies available, surface-enhanced laser desorption/ionization time-of-flight mass spectrometry (SELDI-TOF MS) and matrix-assisted laser desorption/ionization time-of-flight mass spectrometry (MALDI-TOF MS) are attractive techniques since they provide protein profiles in an easy and rapid manner from small amounts of protein, obtained from various biologically complex samples. Younossi et $\mathrm{al}^{100}$ investigated, by means of SELDI-TOF, serum protein profiles of different subtypes of NAFLD and identified 12 significantly different protein peaks across the study groups. Using a similar SELDI-TOF technique, Trak-Smayra et al ${ }^{102}$ have searched for serum markers of steatosis and NASH in obese nonalcoholic patient $(n=80)$ candidates for bariatric surgery. Twenty-four obese patients (30\%) had non-significant liver lesions, 32 (40\%) had significant steatosis, and 24 (30\%) had NASH. Comparison of serum protein profiles according to liver lesions identified three peaks (CM107558.4, CM10-7924.2, and Q10-7926.9), the intensity of which significantly increased according to the severity of the liver lesions (steatosis and NASH) and returned to normal after bariatric surgery. The future availability of rapid, high-throughput analytical platforms is likely to facilitate molecular phenotyping of different subtypes (simple fatty liver, borderline NASH, definitive NASH) in the spectrum of NAFLD. In addition, innovative methods like proteomics and glycomics will be helpful to establish fibrosis specific serum protein and glycosylation patterns, respectively, for diagnosis and monitoring of fibrogenesis in patients with NAFLD.

\section{CONCLUSION}

Currently, the standard procedure for evaluating the degree of necroinflammation and fibrosis, and for quantifying hepatic steatosis remains liver biopsy. However, this is an invasive procedure with unavoidable risks and limitations like sampling error and inter- and intra-observer variations. However, in most cases of NAFLD, the results 
of liver biopsy are not relevant to the choice of treatment, which remains that of metabolic syndrome; moreover, it is difficult to repeat the biopsy after treatment and monitoring the disease progress. Hence, there is an impending need for non-invasive strategies to cover the whole spectrum of NAFLD. Non-invasive investigations, such as various biomarkers, fibrosis scoring panels, and imaging techniques, offer considerable promise in their ability to differentiate simple steatosis from significant fibrosis and to stage liver fibrosis in $80-90 \%$ of cases. So, in day-to-day clinical practice, a liver biopsy is needed only in $10-20 \%$ of patients more for documentation of liver fibrosis rather than making any substantial change in therapeutic intervention. With the advent of proteomics, metabolomics, and better biomarkers studies coupled with promising functional MRS studies, a day may not be far away when liver biopsy may be obsolete at least in distinguishing between simple staetosis and clinically significant fibrosis. Further testing and validation are needed for these noninvasive procedures to refine their role in clinical practice.

\section{CONFLICTS OF INTEREST}

All authors have none to declare.

\section{REFERENCES}

1. Browning JD, Szczepaniak LS, Dobbins R, et al. Prevalence of hepatic steatosis in an urban population in the United States: impact of ethnicity. Hepatology 2004;40:1387-95.

2. Bedogni G, Miglioli L, Masutti F, Tiribelli C, Marchesini G, Bellentani S. Prevalence of and risk factors for non-alcoholic fatty liver disease: the Dionysos nutrition and liver study. Hepatology 2005;42:44-52.

3. Machado M, Marques-Vidal P, Cortez-Pinto H. Hepatic histology in obese patients undergoing bariatric surgery. J Hepatol 2006;45: 600-6.

4. Tominaga K, Kurata JH, Chen YK, et al. Prevalence of fatty liver in Japanese children and relationship to obesity. An epidemiological ultrasonographic survey. Dig Dis Sci 1995;40:2002-9.

5. Franzese A, Vajro P, Argenziano A, et al. Liver involvement in obese children. Ultrasonography and liver enzyme levels at diagnosis and during follow-up in an Italian population. Dig Dis Sci 1997; 42:1428-32.

6. Adams LA, Lymp JF, St Sauver J, et al. The natural history of nonalcoholic fatty liver disease: a population-based cohort study. Gastroenterology 2005;129:113-21.

7. Matteoni CA, Younossi ZM, Gramlich T, Boparai N, Liu YC, McCullough AJ. Nonalcoholic fatty liver disease: a spectrum of clinical and pathological severity. Gastroenterology 1999;116: 1413-9.

8. Angulo P. Long-term mortality in nonalcoholic fatty liver disease: is liver histology of any prognostic significance? Hepatology 2010;51:373-5.

9. Day CP. Natural history of NAFLD: remarkably benign in the absence of cirrhosis. Gastroenterology 2005;129:375-8.

10. Teli MR, James OF, Burt AD, Bennett MK, Day CP. The natural history of nonalcoholic fatty liver: a follow-up study. Hepatology 1995;22:1714-9.

11. Dam-Larsen S, Becker U, Franzmann MB, Larsen K, Christoffersen $P$, Bendtsen F. Final results of a long-term, clinical follow-up in fatty liver patients. Scand J Gastroenterol 2009;44:1236-43.
12. Wong VW, Wong GL, Choi PC, et al. Disease progression of nonalcoholic fatty liver disease: a prospective study with paired liver biopsies at 3 years. Gut 2010;59:969-74.

13. Ekstedt M, Franzen LE, Mathiesen UL, et al. Long-term follow-up of patients with NAFLD and elevated liver enzymes. Hepatology 2006;44:865-73.

14. Adams LA, Sanderson S, Lindor KD, Angulo P. The histological course of nonalcoholic fatty liver disease: a longitudinal study of 103 patients with sequential liver biopsies. J Hepatol 2005;42: 132-8.

15. Starley BQ, Calcagno CJ, Harrison SA. Nonalcoholic fatty liver disease and hepatocellular carcinoma: a weighty connection. Hepatology 2010;51:1820-32.

16. Costera L, Negre I, Samii K, Buffet C. Pain experienced during percutaneous liver biopsy. Hepatology 1999;30:1529-30.

17. Piccinino F, Sagnelli E, Pasquale G, Giusti G. Complications following percutaneous liver biopsy. A multicentre retrospective study on 68,276 biopsies. J Hepatol 1986;2:165-73.

18. Ratziu V, Charlotte F, Heurtier A, et al. Sampling variability of liver biopsy in nonalcoholic fatty liver disease. Gastroenterology 2005; 128:1898-906.

19. Merriman RB, Ferrell LD, Patti MG, et al. Correlation of paired liver biopsies in morbidly obese patients with suspected nonalcoholic fatty liver disease. Hepatology 2006;44:874-80.

20. Cadranel JF, Rufat P, Degos F. Practices of liver biopsy in France: results of a prospective nationwide survey. For the Group of Epidemiology of the French Association for the Study of the Liver (AFEF). Hepatology 2000;32:477-81.

21. Bedossa P, Dargère D, Paradis V. Sampling variability of liver fibrosis in chronic hepatitis C. Hepatology 2003;38:1449-57.

22. Mofrad P, Contos MJ, Haque M, et al. Clinical and histologic spectrum of nonalcoholic fatty liver disease associated with normal ALT values. Hepatology 2003;37:1286-92.

23. Fracanzani AL, Valenti L, Bugianesi E, et al. Risk of severe liver disease in nonalcoholic fatty liver disease with normal aminotransferase levels: a role for insulin resistance and diabetes. Hepatology 2008;48:792-8.

24. Angulo P, Keach JC, Batts KP, Lindor KD. Independent predictors of liver fibrosis in patients with non-alcoholic steatohepatitis. Hepatology 1999;30:1356-62.

25. Ruffillo G, Fassio E, Alvarez E, et al. Comparison of NAFLD fibrosis score and BARD score in predicting fibrosis in nonalcoholic fatty liver disease. J Hepatol 2011;54:160-3.

26. McPherson S, Stewart SF, Henderson E, Burt AD, Day CP. Simple non-invasive fibrosis scoring systems can reliably exclude advanced fibrosis in patients with non-alcoholic fatty liver disease. Gut 2010;59:1265-9.

27. Tahan V, Canbakan B, Balci H, et al. Serum (gamma-glutamyltranspeptidase) distinguishes non-alcoholic fatty liver disease at high risk. Hepatogastroenterology 2008;55:1433-8.

28. Shaheen AA, Myers RP. Diagnostic accuracy of the aspartate aminotransferase-to-platelet ratio index for the prediction of hepatitis C-related fibrosis: a systematic review. Hepatology 2007;46: 912-21.

29. Cales P, Laine F, Boursier J, et al. Comparison of blood tests for liver fibrosis specific or not to NAFLD. J Hepatol 2009;50:165-73.

30. Wai CT, Greenson JK, Fontana RJ, et al. A simple noninvasive index can predict both significant fibrosis and cirrhosis in patients with chronic hepatitis C. Hepatology 2003;38:518-26.

31. Loaeza-del-Castillo A, Paz-Pineda F, Oviedo-Cardenas E, SanchezAvila F, Vargas-Vorackova F. AST to platelet ratio index (APRI) for the noninvasive evaluation of liver fibrosis. Ann Hepatol 2008;7:350-7.

32. Fujii $\mathrm{H}$, Enomoto $\mathrm{M}$, Fukushima W, et al. Noninvasive laboratory tests proposed for predicting cirrhosis in patients with chronic hepatitis $\mathrm{C}$ are also useful in patients with non-alcoholic steatohepatitis. J Gastroenterol 2009;44:608-14. 
33. Manousou P, Kalambokis G, Grillo F, et al. Serum ferritin is a discriminant marker for both fibrosis and inflammation in histologically proven non-alcoholic fatty liver disease patients. Liver Int 2011;31:730-9.

34. Kowdley KV, Belt P, Wilson LA, et al. Serum ferritin is an independent predictor of histologic severity and advanced fibrosis in patients with nonalcoholic fatty liver disease. Hepatology 2012;55:77-85.

35. Sanyal AJ. American Gastroenterological Associatio. AGA technical review on nonalcoholic fatty liver disease. Gastroenterology 2002;123:1705-25.

36. Falck-Ytter Y, Younossi ZM, Marchesini G, McCullough AJ. Clinical features and natural history of nonalcoholic steatosis syndromes. Semin Liver Dis 2001;21:17-26.

37. Mishra P, Younossi ZM. Abdominal ultrasound for diagnosis of nonalcoholic fatty liver disease (NAFLD). Am J Gastroenterol 2007; 102:2716-7.

38. Strauss S, Gavish E, Gottlieb P, Katsnelson L. Interobserver and intraobserver variability in the sonographic assessment of fatty liver. AJR Am J Roentgenol 2007;189:W320-3.

39. Tarantino G, Conca P, Pasanisi F, et al. Could inflammatory markers help diagnose nonalcoholic steatohepatitis? Eur J Gastroenterol Hepatol 2009;21:504-11.

40. lijima H, Moriyasu F, Tsuchiya K, et al. Decrease in accumulation of ultrasound contrast microbubbles in non-alcoholic steatohepatitis. Hepatol Res 2007;37:722-30.

41. Davidson LE, Kuk JL, Church TS, Ross R. Protocol for measurement of liver fat by computed tomography. J Appl Physiol 2006; 100:864-8.

42. Park SH, Kim PN, Kim KW, et al. Macrovesicular hepatic steatosis in living liver donors: use of $\mathrm{CT}$ for quantitative and qualitative assessment. Radiology 2006;239:105-12.

43. Szczepaniak LS, Nurenberg P, Leonard D, et al. Magnetic resonance spectroscopy to measure hepatic triglyceride content: prevalence of hepatic steatosis in the general population. Am J Physiol Endocrinol Metab 2005;288:E462-8.

44. van Werven JR, Hoogduin JM, Nederveen AJ, et al. Reproducibility of 3 Tesla magnetic resonance spectroscopy for measuring hepatic fat content. J Magn Reson Imaging 2009;30:444-8.

45. Guiu B, Petit JM, Loffroy R, et al. Quantification of liver fat content: comparison of triple-echo chemical shift gradient-echo imaging and in vivo proton MR spectroscopy. Radiology 2009;250: 95-102.

46. Yin M, Talwalkar JA, Glaser KJ, et al. Assessment of hepatic fibrosis with magnetic resonance elastography. Clin Gastroenterol Hepatol 2007;5:1207-13.e2.

47. Huwart L, Sempoux C, Vicaut E, et al. Magnetic resonance elastography for the noninvasive staging of liver fibrosis. Gastroenterology 2008;135:32-40.

48. Boursier J, de Ledinghen V, Zarski JP, et al. A new combination of blood test and fibroscan for accurate non-invasive diagnosis of liver fibrosis stages in chronic hepatitis C. Am J Gastroenterol 2011;106:1255-63.

49. Osakabe K, Ichino N, Nishikawa T, et al. Reduction of liver stiffness by antiviral therapy in chronic hepatitis B. J Gastroenterol 2011;46:1324-34.

50. Wong VW, Chu WC, Wong GL, et al. Prevalence of non-alcoholic fatty liver disease and advanced fibrosis in Hong Kong Chinese: a population study using proton-magnetic resonance spectroscopy and transient elastography. Gut 2012;61:409-15 [Epub 2011 Aug 16].

51. Wong VW, Vergniol J, Wong GL, et al. Diagnosis of fibrosis and cirrhosis using liver stiffness measurement in nonalcoholic fatty liver disease. Hepatology 2010;51:454-62.

52. de Lédinghen V, Vergniol J, Foucher J, El-Hajbi F, Merrouche W, Rigalleau V. Feasibility of liver transient elastography with FibroScan using a new probe for obese patients. Liver Int 2010; 30:1043-8.
53. Yoneda M, Yoneda M, Fujita K, et al. Transient elastography in patients with non-alcoholic fatty liver disease (NAFLD). Gut 2007; 56:1330-1.

54. Musso G, Gambino R, Cassader M, Pagano G. Meta-analysis: natural history of non-alcoholic fatty liver disease (NAFLD) and diagnostic accuracy of non-invasive tests for liver disease severity. Ann Med 2011;43:617-49.

55. Castéra L, Vergniol J, Foucher J, et al. Prospective comparison of transient elastography, Fibrotest, APRI, and liver biopsy for the assessment of fibrosis in chronic hepatitis C. Gastroenterology 2005;128:343-50.

56. Harrison SA, Oliver D, Arnold HL, Gogia S, Neuschwander-Tetri BA. Development and validation of a simple NAFLD clinical scoring system for identifying patients without advanced disease. Gut 2008; 57:1441-7.

57. Raszeja-Wyszomirska J, Szymanik B, Lawniczak M, et al. Validation of the BARD scoring system in Polish patients with nonalcoholic fatty liver disease (NAFLD). BMC Gastroenterol 2010;10:67.

58. Fujii $H$, Enomoto $M$, Fukushima W, Tamori A, Sakaguchi $H$, Kawada N. Applicability of BARD score to Japanese patients with NAFLD. Gut 2009;58:1566-7 [Author reply 1567].

59. Yang HR, Kim HR, Kim MJ, Ko JS, Seo JK. Noninvasive parameters and hepatic fibrosis scores in children with nonalcoholic fatty liver disease. World J Gastroenterol 2012;18:1525-30.

60. Shah AG, Lydecker A, Murray K, Tetri BN, Contos MJ, Sanyal AJ. Comparison of noninvasive markers of fibrosis in patients with nonalcoholic fatty liver disease. Clin Gastroenterol Hepatol 2009; 7:1104-12.

61. Sumida $\mathrm{Y}$, Yoneda M, Hyogo $\mathrm{H}$, et al. Validation of the FIB4 index in a Japanese nonalcoholic fatty liver disease population. BMC Gastroenterol 2012;12:2.

62. Cales P, Oberti F, Michalak S, et al. A novel panel of blood markers to assess the degree of liver fibrosis. Hepatology 2005;42:1373-81.

63. Calès $P$, de Ledinghen $V$, Halfon $P$, et al. Evaluating the accuracy and increasing the reliable diagnosis rate of blood tests for liver fibrosis in chronic hepatitis C. Liver Int 2008;28:1352-62.

64. Armstrong MJ, Houlihan DD, Bentham L, et al. Presence and severity of non-alcoholic fatty liver disease in a large prospective primary care cohort. J Hepatol 2012;56:234-40.

65. Ratziu V, Giral P, Charlotte F, et al. Liver fibrosis in overweight patients. Gastroenterology 2000;118:1117-23.

66. Shaheen AA, Wan AF, Myers RP. FibroTest and FibroScan for the prediction of hepatitis C-related fibrosis: a systematic review of diagnostic test accuracy. Am J Gastroenterol 2007;102: 2589-600.

67. Poynard T, Morra R, Halfon P, et al. Meta-analyses of FibroTest diagnostic value in chronic liver disease. BMC Gastroenterol 2007; $7: 40$.

68. Munteanu M, Ratziu V, Morra R, Messous D, Imbert-Bismut F, Poynard T. Noninvasive biomarkers for the screening of fibrosis, steatosis and steatohepatitis in patients with metabolic risk factors: FibroTest-FibroMax experience. J Gastrointest Liver Dis 2008;17: 187-91.

69. Morra R, Munteanu M, Imbert-Bismut F, Messous D, Ratziu V, Poynard T. FibroMAX: towards a new universal biomarker of liver disease? Expert Rev Mol Diagn 2007;7:481-90.

70. Dixon JB, Bhathal PS, O'Brien PE. Nonalcoholic fatty liver disease: predictors of nonalcoholic steatohepatitis and liver fibrosis in the severely obese. Gastroenterology 2001;121:91-100.

71. Palekar NA, Naus R, Larson SP, Ward J, Harrison SA. Clinical model for distinguishing nonalcoholic steatohepatitis from simple steatosis in patients with nonalcoholic fatty liver disease. Liver Int 2006;26:151-6.

72. Gholam PM, Flancbaum L, Machan JT, Charney DA, Kotler DP. Nonalcoholic fatty liver disease in severely obese subjects. Am J Gastroenterol 2007;102:399-408. 
73. Poynard T, Ratziu V, Charlotte F, et al. Diagnostic value of biochemical markers (NashTest) for the prediction of non alcoholo steato hepatitis in patients with non-alcoholic fatty liver disease. BMC Gastroenterol 2006;6:34.

74. Suzuki A, Angulo P, Lymp J, Li D, Satomura S, Lindor K. Hyaluronic acid, an accurate serum marker for severe hepatic fibrosis in patients with non-alcoholic fatty liver disease. Liver Int 2005;25: 779-86.

75. Rosenberg WM, Voelker M, Thiel R, et al. Serum markers detect the presence of liver fibrosis: a cohort study. Gastroenterology 2004;127:1704-13.

76. Guha IN, Parkes J, Roderick P, et al. Noninvasive markers of fibrosis in non-alcoholic fatty liver disease: validating the European Liver Fibrosis Panel and exploring simple markers. Hepatology 2008;47:455-60.

77. Parkes J, Roderick P, Harris S, et al. Enhanced liver fibrosis test can predict clinical outcomes in patients with chronic liver disease. Gut 2010;59:1245-51.

78. Hui JM, Hodge A, Farrell GC, Kench JG, Kriketos A, George J. Beyond insulin resistance in NASH: TNF-alpha or adiponectin? Hepatology 2004;40:46-54.

79. Lemoine M, Ratziu V, Kim M, et al. Serum adipokine levels predictive of liver injury in nonalcoholic fatty liver disease. Liver Int 2009; 29:1431-8.

80. Feldstein AE, Wieckowska A, Lopez AR, Liu YC, Zein NN, McCullough AJ. Cytokeratin-18 fragment levels as noninvasive biomarkers for nonalcoholic steatohepatitis: a multicenter validation study Hepatology 2009;50:1072-8.

81. Younossi ZM, Jarrar M, Nugent C, et al. A novel diagnostic biomarker panel for obesity-related non-alcoholic steatohepatitis (NASH). Obes Surg 2008;18:1430-7.

82. Younossi ZM, Page S, Rafiq N, et al. A biomarker panel for nonalcoholic steatohepatitis (NASH) and NASH-related fibrosis. Obes Surg 2011;21:431-9.

83. Sakugawa H, Nakayoshi T, Kobashigawa K, et al. Clinical usefulness of biochemical markers of liver fibrosis in patients with nonalcoholic fatty liver disease. World J Gastroenterol 2005;11:255-9.

84. Kaneda H, Hashimoto E, Yatsuji S, Tokushige $\mathrm{K}$, Shiratori $\mathrm{K}$. Hyaluronic acid levels can predict severe fibrosis and platelet counts can predict cirrhosis in patients with nonalcoholic fatty liver disease. J Gastroenterol Hepatol 2006;21:1459-65.

85. Lesmana CR, Hasan I, Budihusodo U, et al. Diagnostic value of a group of biochemical markers of liver fibrosis in patients with non-alcoholic steatohepatitis. J Dig Dis 2009;10:201-6.

86. Yoneda M, Yoneda M, Mawatari H, et al. Noninvasive assessment of liver fibrosis by measurement of stiffness in patients with non-alcoholic fatty liver disease (NAFLD). Dig Liver Dis 2008;40: 371-8.

87. Santos VN, Leite-Mor MM, Kondo M, et al. Serum laminin, type IV collagen and hyaluronan as fibrosis markers in non-alcoholic fatty liver disease. Braz J Med Biol Res 2005;38:747-53.
88. Haukeland JW, Damås JK, Konopski Z, et al. Systemic inflammation in nonalcoholic fatty liver disease is characterized by elevated levels of CCL2. J Hepatol 2006;44:1167-74 [Epub 2006 Mar 20].

89. Hui JM, Farrell GC, Kench JG, George J. High sensitivity C-reactive protein values do not reliably predict the severity of histological changes in NAFLD. Hepatology 2004;39:1458-9.

90. Tarantino G, Conca P, Pasanisi F, et al. Could inflammatory markers help diagnose nonalcoholic steatohepatitis? Eur J Gastroenterol Hepatol 2009;21:504-11.

91. Sumida Y, Nakashima T, Yoh T, et al. Serum thioredoxin levels as a predictor of steatohepatitis in patients with nonalcoholic fatty liver disease. J Hepatol 2003;38:32-8.

92. Kayadibi H, Gültepe M, Yasar B, et al. Diagnostic value of serum prolidase enzyme activity to predict the liver histological lesions in non-alcoholic fatty liver disease: a surrogate marker to distinguish steatohepatitis from simple steatosis. Dig Dis Sci 2009; 54:1764-71.

93. Charlton M, Angulo P, Chalasani N, et al. Low circulating levels of dehydroepiandrosterone in histologically advanced nonalcoholic fatty liver disease. Hepatology 2008;47:484-92.

94. Tamimi TI, Elgouhari HM, Alkhouri N, et al. An apoptosis panel for nonalcoholic steatohepatitis diagnosis. J Hepatol 2011;54: 1224-9.

95. Yilmaz Y, Ulukaya E, Gul O0, et al. Decreased plasma levels of soluble receptor for advanced glycation endproducts (SRAGE) in patients with nonalcoholic fatty liver disease. Clin Biochem 2009;42:802-7.

96. Alkhouri N, Lopez R, Berk M, Feldstein AE. Serum retinolbinding protein 4 levels in patients with nonalcoholic fatty liver disease. J Clin Gastroenterol 2009;43:985-9.

97. Degertekin B, Ozenirler S, Elbeg S, Akyol G. The serum endothelin-1 level in steatosis and NASH, and its relation with severity of liver fibrosis. Dig Dis Sci 2007;52:2622-8.

98. Yilmaz, Y, Ulukaya E, Atug O, Dolar E. Serum concentrations of human angiopoietin-like protein 3 in patients with nonalcoholic fatty liver disease: association with insulin resistance. Eur J Gastroenterol Hepatol 2009;21:1247-51.

99. Kukla M, Zwirska-Korczala K, Hartleb M, et al. Serum chemerin and vaspin in non-alcoholic fatty liver disease. Scand $J$ Gastroenterol 2010;45:235-42.

100. Younossi ZM, Baranova A, Ziegler K, et al. A genomic and proteomic study of the spectrum of nonalcoholic fatty liver disease. Hepatology 2005;42:665-74.

101. Wieckowska A, McCullough AJ, Feldstein AE. Noninvasive diagnosis and monitoring of nonalcoholic steatohepatitis: present and future. Hepatology 2007;46:582-9.

102. Trak-Smayra V, Dargere D, Noun R, et al. Serum proteomic profiling of obese patients: correlation with liver pathology and evolution after bariatric surgery. Gut 2009;58:825-32 [Epub 2008 Apr 10]. 